Laurence Senn

Philippe Eggimann

Riadh Ksontini

Andres Pascual

Nicolas Demartines

Jacques Bille

Thierry Calandra

Oscar Marchetti

\section{Caspofungin for prevention of intra-abdominal candidiasis in high-risk surgical patients}

Received: 17 June 2008

Accepted: 30 December 2008

Published online: 27 January 2009

(C) Springer-Verlag 2009

This study has been partially presented as poster \# M-1163 at the 47th ICAAC

(Interscience Conference on Antimicrobial

Agents and Chemotherapy), Chicago, 17-20

September 2007.

L. Senn · A. Pascual · J. Bille .

T. Calandra $\cdot$ O. Marchetti ( $)$

Infectious Diseases Service,

Department of Medicine,

Centre Hospitalier Universitaire Vaudois

(CHUV) and University of Lausanne

(UNIL), Rue du Bugnon 46,

1011 Lausanne, Switzerland

e-mail: Oscar.Marchetti@chuv.ch

Tel.: +41-21-3141010

Fax: +41-21-3141018

P. Eggimann

Adult Intensive Care Service,

Centre Hospitalier Universitaire Vaudois

(CHUV) and University of Lausanne

(UNIL), Lausanne, Switzerland

R. Ksontini · N. Demartines

Visceral Surgery Service,

Centre Hospitalier Universitaire Vaudois

(CHUV) and University of Lausanne

(UNIL), Lausanne, Switzerland
J. Bille

Institute of Microbiology, Centre

Hospitalier Universitaire Vaudois (CHUV)

and University of Lausanne (UNIL),

Lausanne, Switzerland

Abstract Purpose: Thirty to forty percent of patients with recurrent gastrointestinal perforation/anastomotic leakage or acute necrotizing pancreatitis develop intra-abdominal invasive candidiasis (IC). A corrected Candida colonization index (CCI) $\geq 0.4$ is a powerful predictor of IC. Fluconazole prevents intra-abdominal IC in this setting, but azole-resistant Candida species are emerging. The aim of this study was to explore the efficacy and safety of caspofungin for prevention of intra-abdominal IC in high-risk surgical patients.

Methods: Prospective non-comparative single-center study in consecutive adult surgical patients with recurrent gastrointestinal perforation/anastomotic leakage or acute necrotizing pancreatitis. Preventive caspofungin therapy $(70 \mathrm{mg}$, then $50 \mathrm{mg} /$ day) was given until resolution of the surgical condition. Candida colonization index and CCI, occurrence of intra-abdominal IC and adverse events were monitored. Results: Nineteen patients were studied: 16 (84\%) had recurrent gastrointestinal perforation/anastomotic leakage and $3(16 \%)$ acute necrotizing pancreatitis. The median duration of preventive caspofungin therapy was 16 days (range 4-46). The colonization index decreased significantly during study therapy, and the CCI remained $<0.4$ in all patients. Caspofungin was successful for prevention of intra-abdominal IC in 18/19 patients $(95 \%, 1$ breakthrough IC 5 days after inclusion). No drugrelated adverse event requiring caspofungin discontinuation occurred. Conclusion: Caspofungin may be efficacious and safe for prevention of intra-abdominal candidiasis in highrisk surgical patients. This needs to be further investigated in randomized trials.

Keywords Caspofungin . Prevention - Surgery - Pancreatitis . Candidiasis · Fungal infection

\section{Background}

Invasive candidiasis (IC) has emerged as a frequent complication in surgical critically ill patients $[1,2]$. About one-third of patients with recurrent gastrointestinal perforations, anastomotic leakages or acute necrotizing pancreatitis develop intra-abdominal IC [3, 4]. Candida colonization is a major risk factor for IC in this setting 
[5-7], and a corrected colonization index $(\mathrm{CCI}) \geq 0.4$ has been shown to predict IC with an efficiency of $100 \%$ [8].

Early identification of non-neutropenic patients at high risk of IC remains difficult and late therapy is associated with high morbidity and mortality. Few data are available on the utility of monitoring Candida colonization and/or non-invasive fungal markers [9-13]. Clinical prediction rules of the risk of IC have been investigated, but their role for guiding the decision to start antifungal therapy remains to be established [14, 15]. Fluconazole has been shown to prevent IC in high-risk surgical patients with a number needed to treat ranging between 3 and $10[3,12$, 16-20]. In contrast, a study in ICU patients with persistent fever despite antibacterial therapy (incidence of IC 9\%) failed to demonstrate the efficacy of empirical fluconazole therapy [21]. Based on a moderate to high level of evidence, guidelines from the Infectious Diseases Society of America (IDSA) recommend fluconazole for prevention of IC in high-risk surgical ICU settings $[20,22]$.

A broad use of azoles has been associated with a shift towards infections due to non-albicans Candida species with reduced antifungal susceptibility [23-26]. Preventive antifungal therapy should thus be used very selectively in patients at highest risk. Caspofungin, an approved agent for therapy of mucosal and invasive candidiasis, is active against azole-resistant Candida spp. [27]. As no experience on the role of echinocandins for prevention of intraabdominal candidiasis in high-risk surgical patients is available, the aim of this study was to explore the efficacy and safety of caspofungin in this setting.

\section{Patients and methods}

Design

We conducted a prospective non-comparative singlecenter study between February 2006 and March 2007. The study was approved by the institutional ethical committee.

\section{Patients' selection}

Inclusion criteria were: (1) age $\geq 16$ years, (2) recurrent gastrointestinal perforations/anastomotic leakage(s) after abdominal surgery or surgery for acute necrotizing pancreatitis during the preceding 7 days, and (3) written informed consent. Exclusion criteria were (1) documented IC at study entry, (2) ongoing antifungal therapy during $>48 \mathrm{~h}$, (3) severe hepatic insufficiency (Child-Pugh score $>9$ ), (4) caspofungin allergy, (5) pregnant or lactating woman, or (6) high probability of death within $72 \mathrm{~h}$.
Preventive caspofungin therapy

A caspofungin loading dose $(70 \mathrm{mg})$, was followed by $50 \mathrm{mg} /$ day until resolution of the surgical condition defined by (1) recovery of gastrointestinal function and (2) no complication requiring surgical reintervention.

\section{Clinical data}

Demographic, clinical and laboratory data were collected at study entry and then twice weekly until end of followup, i.e., 1 week after end of study therapy or death.

\section{Microbiological follow-up}

Oral cavity, stool, urine, peritoneal fluid (at laparotomy or from surgical drains), and additional sites according to the underlying conditions were monitored for Candida colonization. Candida cultures on Candida-specific chromogenic plates $\left(\mathrm{CAN} 2^{\circledR}\right.$, Biomerieux, France) were performed at study entry, twice during the first week, and then once weekly until end of follow-up. Colonization was assessed as weak, moderate, or heavy by semi-quantitative cultures using the clock-streak technique [5]. The colonization index (number of sites colonized/number of sites screened) and the CCI [(number of sites with heavy colonization/number of sites colonized) $\times$ colonization index] were calculated as previously reported [8]. Colonizing species were differentiated as Candida albicans and nonalbicans.

Cultures of blood, urine, peritoneal fluid, surgical wounds, and other sites were performed as clinically indicated in the presence of symptoms/signs of infection. Invasive Candida isolates were identified to the species level and antifungal susceptibility was tested according to CLSI standards [28].

\section{Definitions of Candida infections}

IC was defined by: (1) intra-abdominal candidiasis including (1a) abscess with Candida isolated in pure or mixed culture or (1b) peritonitis in presence of fever, abdominal pain, tenderness on palpation, ileus, and leukocytosis $\left(>10 \times 10^{9} / \mathrm{L}\right)$ with isolation of Candida in the peritoneal fluid from laparotomy or drain effluents (in mixed bacterial and fungal cultures, Candida was regarded as pathogen when recovered from blood or in increasing amounts in serial cultures in absence of clinical improvement despite appropriate surgical and antibacterial treatment), (2) candidemia, i.e., positive blood cultures and clinical symptoms/signs of infection, (3) Candida urinary tract infection, i.e., $>10^{5} \mathrm{CFU} / \mathrm{ml}$ with pyuria and fever without other focus and/or with local symptoms/signs of 
infection, (4) infection at another site, e.g., surgical wound infection with Candida isolated from a biopsy specimen or a purulent discharge.

\section{Endpoints}

The primary endpoint was the absence of breakthrough intra-abdominal IC during and within 1 week after discontinuation of preventive caspofungin therapy. Secondary endpoints were (1) absence of breakthrough extraabdominal IC, (2) survival, (3) Candida colonization indices, and (4) drug-related adverse events according to the National Cancer Institute criteria [29].

\section{Statistics}

No sample size calculation had been performed for this observational non-comparative single-center exploratory study. Continuous variables and proportions of Candida colonization at start and end of preventive therapy were compared by non-parametric paired Wilcoxon signed rank test and Fisher's exact test, respectively. The correlation of continuous variables was studied by the non-parametric Spearman rank test.

\section{Results}

Clinical characteristics

Nineteen patients were included in the study. Demographics and clinical characteristics are shown in Table 1. All patients had abdominal surgery before inclusion: explorative laparotomy with/without gut resection and/or peritoneal lavage, or pancreatic necrosectomy. Fourteen (74\%) patients required additional abdominal surgery after study entry.

\section{Candida colonization}

Data on Candida colonization are summarized in Table 2. Colonization index and CCI at baseline and at end of preventive caspofungin therapy are shown in Fig. 1. No patient developed a $\mathrm{CCI} \geq 0.4$ during preventive therapy. A decline of the colonization index was correlated with the duration of preventive caspofungin therapy (Spearman correlation coefficient $r=-0.46, P=0.049$ ).

Success of preventive caspofungin therapy

Caspofungin was successful for prevention of intraabdominal IC in $18 / 19$ patients $(95 \%)$. Only one
Table 1 Patients demographics and clinical characteristics

\begin{tabular}{ll}
\hline Patients & 19 \\
Gender: male/female & $16 / 3$ \\
Age & $69(40-84)$ \\
Underlying surgical conditions & \\
Gastrointestinal perforation/anastomotic leakage & $16(84 \%)$ \\
Necrotizing pancreatitis & $3(16 \%)$ \\
Number of abdominal surgical interventions ${ }^{\mathrm{a}}$ & \\
Before inclusion & $2(1-6)$ \\
During preventive caspofungin therapy & $1(0-6)$ \\
Antibiotic therapy at baseline & $19(100 \%)$ \\
Hospitalized in ICU & $14(74 \%)$ \\
APACHE ${ }^{\mathrm{b}}$ II score at ICU admission & $17(10-30)$ \\
SAPS II score at ICU admission $_{\text {Mechanical ventilation }}$ & $45(31-65)$ \\
Days of ICU stay & $10(53 \%)$ \\
Before inclusion & $3(0-14)$ \\
Overall & $13(3-56)$ \\
Days of preventive caspofungin therapy & $16(4-46)$ \\
Days of follow-up after study entry & $28(6-182)$ \\
Days of hospital stay & \\
Before inclusion & $10(1-43)$ \\
Overald & $37(17-194)$ \\
\hline
\end{tabular}

Data are median (range), unless specified otherwise

a Explorative laparotomy with/without gut resection and/or peritoneal lavage, or pancreatic necrosectomy for therapy of recurrent gastrointestinal perforations/anastomotic leakage(s) or for acute necrotizing pancreatitis

b Acute physiology and chronic health evaluation

c Simplified acute physiology score

d In 14 patients who survived and were discharged

breakthrough IC (mixed intra-abdominal abscess due to Enterococcus spp., C. glabrata and C. lipolytica) occurred in a patient with ischemic colitis and recurrent intestinal perforations, who died from an intra-abdominal bleeding 5 days after study inclusion (no autopsy performed). However, as no intra-abdominal sampling for culture was available at baseline, an IC ongoing at study entry could not be ruled out. At the time of diagnosis, the $C$. glabrata isolate was susceptible to fluconazole and caspofungin (MIC 8 and $0.16 \mathrm{mg} / \mathrm{l})$ and the $C$. lipolytica isolate had a reduced susceptibility to fluconazole (MIC $16 \mathrm{mg} / \mathrm{l}$ ) and was susceptible to caspofungin (MIC $0.25 \mathrm{mg} / \mathrm{l}$ ). The patient was colonized at study entry and remained colonized at time of death by a non-albicans Candida spp. No extraabdominal breakthrough IC occurred.

\section{Survival}

Fourteen patients (74\%) survived. Causes of death were: (1) acute necrotizing pancreatitis with multiple organ failure, intestinal perforation and bacterial peritonitis $(n=2)$, (2) ischemic colitis with extended intestinal necrosis, multiple perforations and bacterial peritonitis $(n=2)$, and (3) intra-abdominal hemorrhage with concomitant breakthrough IC ( $n=1$, see above). 
Table 2 Candida colonization

\begin{tabular}{lll}
\hline & At study entry & $\begin{array}{c}\text { At end of preventive } \\
\text { therapy }^{\mathrm{a}}\end{array}$ \\
\hline Patients colonized with Candida spp. & $9 / 14(64 \%)^{\mathrm{b}, \mathrm{c}}$ \\
Number of screened sites per patient, median (range) & $4(0-5)$ \\
Number of colonized sites per patient, median (range) & $4(3-5)$ & $1.5(0-3)$ \\
Number of sites colonized/number of sites screened & $2(0-4)$ & $17 / 53(32 \%)^{\mathrm{d}}$ \\
Oropharynx & $34 / 68(50 \%)$ & $5 / 12(42 \%)$ \\
Stools & $13 / 18(72 \%)$ & $7 / 11(64 \%)$ \\
Lower respiratory tract & $7 / 7(100 \%)$ & $2 / 6(33 \%)$ \\
Peritoneal fluid & $7 / 9(78 \%)$ & $3 / 13(23 \%)$ \\
Urine & $6 / 17(35 \%)$ & $0 / 12(0 \%)$ \\
Number of colonizing Candida isolates & $1 / 17(6 \%)$ & $266^{\mathrm{e}}$ \\
Candida albicans & $39^{\mathrm{e}}$ & $19 / 26(73 \%)$ \\
Non-albicans Candida species & $27 / 39(69 \%)$ & $7 / 26(27 \%)$ \\
\hline
\end{tabular}

${ }^{\text {a }}$ In patients who survived

${ }^{c} P=0.11$. In all patients, including those who died: $14 / 19(74 \%)$

b In 1 patient who was not colonized at baseline, colonization with d $P=0.06$. In all patients, including those who died: $29 / 75$ (39\%) a non-albicans Candida species was documented at 1 site (stool) e Four sites with $\geq 2$ Candida species during preventive therapy

Adverse events (AE)

No AE with probable or proven relationship with caspofungin was documented. AE possibly related to caspofungin were observed in 8 patients $(42 \%)$ : cholestasis in $7(37 \%)$ : grade 1 in 3 , grade 2 in 1 , and grade 3 in 3 ; hepatic cytolysis in 2 (32\%), both grade 1; and skin rash in $2(11 \%)$, both grade 1 . However, all these patients were receiving simultaneously multiple co-medications, which may have been implicated in these AE. No AE required discontinuation of caspofungin.

\section{Discussion}

Based on a previous study conducted in our institution, we estimated that the risk of developing intra-abdominal

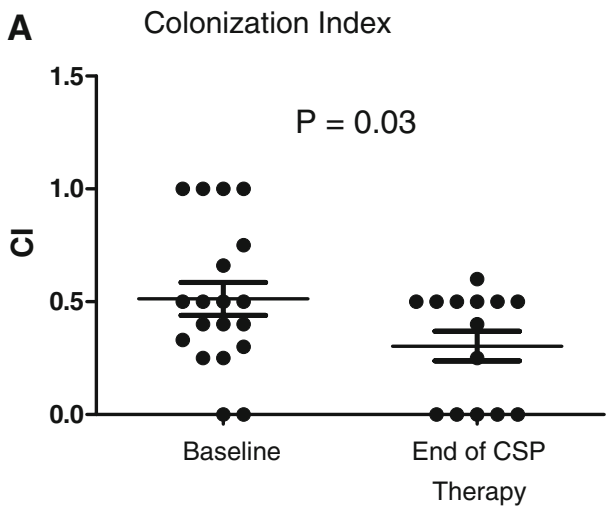

Fig. 1 Semi-quantitative assessment of the Candida colonization at baseline and at end of preventive caspofungin therapy in patients who survived. Panel a colonization index $(\mathrm{CI}=$ number of sites colonized/number of sites screened). Panel $\mathbf{b}$ corrected colonization index $[\mathrm{CCI}=$ (number of sites heavily colonized/number of
IC in patients with recurrent gastrointestinal perforations, anastomotic leakages or acute necrotizing pancreatitis ranges $30-40 \%$ [3]. In this study of preventive caspofungin therapy in surgical patients enrolled on the basis of a high-risk clinical profile before the colonization status was known, this complication occurred in one single case $(5 \%)$. This failure occurred despite in vitro susceptibility of the Candida isolates to caspofungin in a patient with a severe unresolved surgical condition complicated by intra-abdominal bleeding (primary cause of death) and bacterial co-infection, in whom an IC at baseline could not be ruled out. As expected from the drug safety profile, no $\mathrm{AE}$ requiring discontinuation of caspofungin was observed.

As in other studies in surgical patients with similar high-risk profiles $[3,5,8,16,17,30]$, most patients were colonized with Candida at baseline, but none with a CCI exceeding 0.4 , a previously reported powerful predictor of

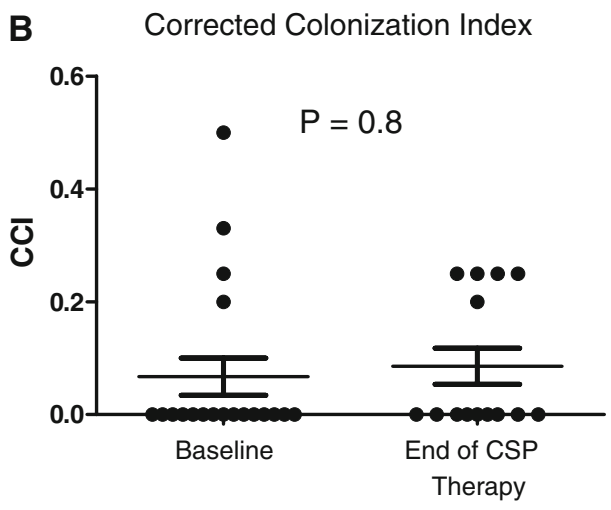

sites colonized $\times$ colonization index]. Each symbol represents a single patient. The horizontal bars represent the median values at each time point. Previous studies have suggested that a CCI $\geq 0.4$ may predict IC with a positive predictive value of $100 \%$ 
IC, which would have made them eligible for pre-emptive antifungal therapy $[8,11]$. The Candida colonization index decreased at end of preventive therapy, and no patient developed a CCI exceeding 0.4. This finding is suggestive of a preventive action of caspofungin on the development of a heavy colonization, one of the initial steps in the pathogenesis of IC. No shift towards nonalbicans Candida species occurred during preventive caspofungin therapy.

No experience is available on the efficacy of echinocandins, a new class of fungicidal drugs active against Candida species with decreased susceptibility to azoles, for prevention of IC. Despite limitations such as the open single-center non-comparative design and the small sample size, the observations of this proof-of-concept study suggest that caspofungin may be efficacious and safe for prevention of intra-abdominal candidiasis in surgical patients with a high-risk profile. This could be of particular interest in hospitals with a high prevalence of azole-resistant Candida species. The present exploratory results provide a stepping stone to future randomized studies designed to define the role of echinocandins for prevention of IC in such a high-risk setting, and to assess its impact on morbidity, mortality, and pharmaco-economic issues. Whether other antifungals licensed for therapy of IC, such as liposomal amphotericin B or voriconazole, may also be efficacious for prevention of IC needs to be investigated. Moreover, the utility of monitoring Candida colonization and of clinical prediction rules for guiding the decision to start pre-emptive antifungal therapy remains to be defined. Waiting for all these additional data, fluconazole remains the recommended first-line choice for a prevention based on straightforward clinical criteria.

Acknowledgments We are thankful to Noémie Calandra, Isabel Cobos, Christian Durussel, Monika Ochsner, Annie Savoie, Aline Wenger, and the staffs of the Laboratories of Mycology and Serology of the Institute of Microbiology and of the Adult Intensive Care and Visceral Surgery Services for the outstanding assistance in collection and management of clinical data and blood samples. The study was supported by an unrestricted research grant of Merck, Sharp and Dohme-Chibret. The sponsor was neither involved in designing and running of the study, in data collection, analysis and interpretation, nor in redaction of the manuscript.

\section{References}

1. Marchetti O, Bille J, Fluckiger U, Eggimann P, Ruef C, Garbino J, Calandra T, Glauser MP, Tauber MG, Pittet D (2004) Epidemiology of candidemia in Swiss tertiary care hospitals: secular trends, 1991-2000. Clin Infect Dis 38:311-320

2. Bougnoux ME, Kac G, Aegerter P, d'Enfert C, Fagon JY (2008)

Candidemia and candiduria in critically ill patients admitted to intensive care units in France: incidence, molecular diversity, management and outcome. Intensive Care Med 34:292-299

3. Eggimann P, Francioli P, Bille J, Schneider R, Wu MM, Chapuis G, Chiolero R, Pannatier A, Schilling J, Geroulanos S, Glauser MP, Calandra T (1999) Fluconazole prophylaxis prevents intra-abdominal candidiasis in high-risk surgical patients. Crit Care Med 27:1066-1072

4. De Waele JJ, Vogelaers D, Blot S, Colardyn F (2003) Fungal infections in patients with severe acute pancreatitis and the use of prophylactic therapy. Clin Infect Dis 37:208-213

5. Calandra T, Bille J, Schneider R, Mosimann F, Francioli P (1989) Clinical significance of Candida isolated from peritoneum in surgical patients. Lancet 2:1437-1440
6. Sandven P, Qvist H, Skovlund E, Giercksky KE (2002) Significance of Candida recovered from intraoperative specimens in patients with intraabdominal perforations. Crit Care Med 30:541-547

7. Calandra T, Marchetti O (2004) Clinical trials of antifungal prophylaxis among patients undergoing surgery. Clin Infect Dis 39(Suppl 4):S185-S192

8. Pittet D, Monod M, Suter PM, Frenk E, Auckenthaler R (1994) Candida colonization and subsequent infections in critically ill surgical patients. Ann Surg 220:751-758

9. Rex JH, Sobel JD (2001) Prophylactic antifungal therapy in the intensive care unit. Clin Infect Dis 32:1191-1200

10. Calandra T, Marchetti O (2002) Antifungal prophylaxis for intensive care unit patients: let's fine tune it. Intensive Care Med 28:1698-1700

11. Piarroux R, Grenouillet F, Balvay $P$, Tran V, Blasco G, Millon L, Boillot A (2004) Assessment of preemptive treatment to prevent severe candidiasis in critically ill surgical patients. Crit Care Med 32:2443-2449

12. Shorr AF, Chung K, Jackson WL, Waterman PE, Kollef MH (2005) Fluconazole prophylaxis in critically ill surgical patients: a meta-analysis. Crit Care Med 33:1928-1935
13. Prella M, Bille J, Pugnale M, Duvoisin B, Cavassini M, Calandra T, Marchetti O (2005) Early diagnosis of invasive candidiasis with mannan antigenemia and antimannan antibodies. Diagn Microbiol Infect Dis 51:95-101

14. Ostrosky-Zeichner L, Sable C, Sobel J, Alexander BD, Donowitz G, Kan V, Kauffman CA, Kett D, Larsen RA, Morrison V, Nucci M, Pappas PG, Bradley ME, Major S, Zimmer L, Wallace D, Dismukes WE, Rex JH (2007) Multicenter retrospective development and validation of a clinical prediction rule for nosocomial invasive candidiasis in the intensive care setting. Eur J Clin Microbiol Infect Dis 26:271276

15. Leon C, Ruiz-Santana S, Saavedra P, Almirante B, Nolla-Salas J, VarezLerma F, Garnacho-Montero J, Leon MA (2006) A bedside scoring system ("Candida score") for early antifungal treatment in nonneutropenic critically ill patients with Candida colonization. Crit Care Med 34:730-737

16. Pelz RK, Hendrix CW, Swoboda SM, Diener-West M, Merz WG, Hammond J, Lipsett PA (2001) Double-blind placebo-controlled trial of fluconazole to prevent candidal infections in critically ill surgical patients. Ann Surg 233:542-548 
17. Garbino J, Lew DP, Romand JA, Hugonnet S, Auckenthaler R, Pittet D (2002) Prevention of severe Candida infections in nonneutropenic, high-risk, critically ill patients: a randomized, double-blind, placebo-controlled trial in patients treated by selective digestive decontamination. Intensive Care Med 28:1708-1717

18. Ho KM, Lipman J, Dobb GJ, Webb SA (2005) The use of prophylactic fluconazole in immunocompetent highrisk surgical patients: a meta-analysis. Crit Care 9:R710-R717

19. Playford EG, Webster AC, Sorrell TC, Craig JC (2006) Antifungal agents for preventing fungal infections in nonneutropenic critically ill and surgical patients: systematic review and metaanalysis of randomized clinical trials. $\mathbf{J}$ Antimicrob Chemother 57:628-638

20. Cruciani M, de Lalla F, Mengoli C (2005) Prophylaxis of Candida infections in adult trauma and surgical intensive care patients: a systematic review and meta-analysis. Intensive Care Med 31:1479-1487

21. Schuster MG, Edwards JE Jr, Sobel JD, Darouiche RO, Karchmer AW, Hadley S, Slotman G, Panzer H, Biswas P, Rex JH (2008) Empirical fluconazole versus placebo for intensive care unit patients: a randomized trial. Ann Intern Med 149:83-90
22. Pappas PG, Rex JH, Sobel JD, Filler SG, Dismukes WE, Walsh TJ, Edwards JE (2004) Guidelines for treatment of candidiasis. Clin Infect Dis 38:161-189

23. Wingard JR, Merz WG, Rinaldi MG, Johnson TR, Karp JE, Saral R (1991) Increase in Candida krusei infection among patients with bone marrow transplantation and neutropenia treated prophylactically with fluconazole. N Engl J Med 325:1274-1277

24. Abi-Said D, Anaissie E, Uzun O, Raad I, Pinzcowski H, Vartivarian S (1997) The epidemiology of hematogenous candidiasis caused by different Candida species. Clin Infect Dis 24:1122-1128

25. Eggimann P, Garbino J, Pittet D (2003) Epidemiology of Candida species infections in critically ill nonimmunosuppressed patients. Lancet Infect Dis 3:685-702

26. Shorr AF, Lazarus DR, Sherner JH, Jackson WL, Morrel M, Fraser VJ, Kollef MH (2007) Do clinical features allow for accurate prediction of fungal pathogenesis in bloodstream infections? Potential implications of the increasing prevalence of non-albicans candidemia. Crit Care Med 35:1077-1083
27. Mora-Duarte J, Betts R, Rotstein C, Colombo AL, Thompson-Moya L, Smietana J, Lupinacci R, Sable C, Kartsonis N, Perfect J (2002) Comparison of caspofungin and amphotericin B for invasive candidiasis. N Engl J Med 347:2020 2029

28. Clinical and Laboratory Standards Institute (2005) Performance standards for antimicrobial susceptibility testing: fifteenth informational supplement. Clinical and laboratory Standards Institute, Wayne, PA

29. Common Terminology Criteria for Adverse Events v3.0 (CTCAE) Published 9 August 2006. http://ctep.cancer.gov/forms/ CTCAEv3.pdf, 2008

30. Charles PE, Dalle F, Aube H, Doise JM, Quenot JP, Aho LS, Chavanet P, Blettery B (2005) Candida spp. colonization significance in critically ill medical patients: a prospective study. Intensive Care Med 31:393-400 